\title{
Spatial orientation during eyes closed versus open in the dark: Are they the same?
}

\author{
Raymond K. Y. Chong*, Ben Gibson, Scott Horton, April Lee, Jordan Mellinger, Kyoung-Hyun Lee
}

Department of Physical Therapy, Georgia Health Sciences University, Augusta, USA

Email: ${ }^{*}$ rchong8@hotmail.com

Received 31 December 2011; revised 9 February 2012; accepted 16 February 2012

\begin{abstract}
We hypothesized that keeping one's balance with eyes open in the dark is different and more difficult than eyes closed because the brain continues to process visual inputs in the dark when the eyes are open. On the other hand, when the eyes are closed, the visual system does not signal incongruent information with which the brain must compare the other sensory systems. A variety of cognitive (subtracting backwards by seven as quickly and accurately as possible) and support surface (fixed versus sway-referenced) conditions were used to probe the neural mechanisms underlying the sensory organization processes in healthy young adults. Peak-to-peak anteroposterior sway performance revealed two dissociated components of the treatment effects. The first component came from the visuospatial factor. Balance control during eye closure and eyes open in the dark were found to be similar but poorer than baseline condition (eyes open under typical lighting). The second component was the effect of task difficulty in which balance control in the sway-referenced condition was worse compared to fixed support during eye closure or eyes open in the dark. Analyses of the cognitive performance also revealed different underlying neural mechanisms of the experimental conditions. Subtraction speed under the fixed support surface condition was similar among all the conditions but was faster with eyes closed during the sway-referenced support surface condition. Accuracy was not affected among the visual and surface conditions. We conclude that sensory processing load with eyes closed is lower than eyes open in the dark, thereby allowing cognitive performance to proceed more efficiently. Performing a difficult subtraction task with eyes closed may afford a decrease in dual-task interference since similar brain areas, particularly the parietal region, are involved in both tasks. The results are discussed with reference to clinical application and spatial disorientation in aviation.
\end{abstract}

*Corresponding author.
Keywords: Attention; Balance; Computational

Neuroscience; Dual-Task; Modular Theory; Parietal;

Romberg; Visuospatial

\section{INTRODUCTION}

Our study was designed to explore several aspects of stance balance control in the dark and how it might be affected by: 1) the search for visual cues, 2) the lack of visual cues, and 3) multi-tasking the area of the brain that is responsible for visuospatial processing and how they might applied to a better understanding of spatial disorientation in aviation. According to the theory of modularity of brain function, certain brain areas are specialized to perform specific computational functions $[1,2]$. Motor and/or cognitive activities that contain similar computational processes utilize the same brain area(s) to fulfill those functions $[3,4]$. A logical prediction of the theory therefore is that a computational overload or interference is expected to occur if two or more tasks that call upon similar computations are performed concurrently [5].

In the analyses of performance interference between two tasks, our interest lies in what is referred to as an internal structural interference [6]. By internal we mean the performance costs that arise when the same area of the brain has to deal with the simultaneous processing of the sub-components of the two tasks. In contrast, external structural interference relates to using the same body part to carry out two tasks, in which case the root cause of the majority of the performance costs can readily be accounted for. One example of such interference is texting while driving. Here, the eyes must focus on both the road and the texting device. Likewise, the hands must type while they are steering the vehicle. The physical constraints of attempting to do both tasks concurrently are clearly so substantial that is very difficult to account for the component of performance decrement that comes from central sources, i.e. diversion of attention.

Such experimental confounds may be avoided when studying the mechanisms of internal interferences by 
selecting two tasks that are as physically independent from each other as possible. In a previous study, we paired a stance balance control task with either an arithmetic subtraction task or a word generation task [6]. Balance control is a complex visuospatial task which involves the interaction of dynamic sensorimotor processes that include biomechanical constraints, movement strategies, sensory integration, orientation in space, cognitive processing, experience and practice, and perception of goal and context [7-10]. Mental arithmetic computations such as subtracting backwards by seven [11] also activate visuospatial areas of the brain including the bilateral inferior parietal region. Performance costs were found in the first pairing of tasks [6]. The more demanding the visuospatial (balance control) conditions, the more the interference between the two tasks was observed.

The current study extends these findings by exploring the visuospatial significance of keeping the eyes open while standing in total darkness. Monkey studies have shown that with the eyes fixated on a target, half of the so-called visual neurons in the posterior parietal area (V6), which receive inputs from the occipital region, were found to be active under total darkness condition [12]. These neurons are thought to be involved in general motor control rather than eye movements [13]. In human studies, the right posterior inferior parietal region, among other areas, was found to be active when the eyes are open in the dark compared to eyes closed [14]. Certain brain areas were also active during eye closure. Other than the somatosensory cortex, however, the parietal region remained relatively quiet. The primary visual cortex is silent when the eyes are open and moving in the dark [15], so it is not the source of parietal activation. Eye blinking per se also does not activate the posterior parietal region [16] unless it is induced artificially via electrical stimulation [17].

Eliminating visual inputs via eye closure is thought to improve the visuospatial mechanisms for balance control [18] and/or cognitive performance compared to eyes open in darkness. If so, this may explain why people sometimes close their eyes in order to process a response - it seems to help us visualize our thoughts better [19]. We hypothesize that the potential benefits of eye closure are specific to activities that interfere with each other. In the case of the subtraction task, difficult arithmetic problems seem to cause the brain to visually imagine the computations [20]. This transforms what appears to be an arithmetic task into a visuospatial task, thereby accounting for the parietal activation. The results of the current study showed that eye closure produced better subtraction performance during a concomitant standing balance control task, concurring with our postulation that eye closure reduces the visuospatial interference between the two tasks.

\section{MATERIALS AND METHODS}

\subsection{Participants}

A convenience sample of 50 subjects (24 men and 26 women, $24 \pm 2$ years old) who reported no significant musculoskeletal or neurological impairment participated in the study approved by the institutional review board.

\subsection{Common Procedures}

There were twelve visuospatial conditions: two distraction, two support, and three vision conditions. Visuospatial performance was quantified using the Sensory Organization Test (SOT) routine (NeuroCom International Inc., Clackamas, Oregon). Subjects stood on a dual force plate inside a 3-sided visual-surround, looking forward, arms by the side, and ankle joints positioned over the rotational axis of the force plates $(100 \mathrm{~Hz}$ sampling rate). In some conditions, the support surface floor plates were sway-referenced, i.e. they rotated toes-up or toes-down in proportion to the subject's anteroposterior body sway. These two support conditions were mixed with three vision conditions: 1) eyes open with lights on (EoOn), 2) eyes open in total darkness (EoOff), and 3) eyes closed with lights on (EcOn). In all twelve conditions, the subjects were instructed not to move their feet or reach out to touch the visual surround. Figure 1 illustrates these conditions. Each condition was tested thrice consecutively. Each trial lasted $20 \mathrm{~s}$ with a pause of $10-20 \mathrm{~s}$ between trials. Subjects wore comfortable flat shoes and were fitted with a safety harness during testing which allowed free movements but would prevent an actual fall.

\subsection{Retro-7 (Subtraction) Protocol}

Subjects were randomized to either the Retro-7 subtraction group or the Control (no subtraction) group. The groups then switched protocol a month later to complete the second session of testing in the order of conditions that mirrored their first session. Within each group, subjects were further randomized into two sub-groups. Both groups underwent the EoOn condition first. The first subgroup was then tested with EcOn followed by EoOff while the second sub-group was tested in the reverse order. To achieve the maximum distraction during the tests, subjects were instructed to subtract a random three-digit number aloud as quickly and as accurately as possible.

\subsection{Analyses}

Balance control performance was quantified using the equilibrium score (ES) which is the peak-to-peak anteroposterior (AP) body center of mass sway recorded in each trial. The formula used to calculate the equilibrium 


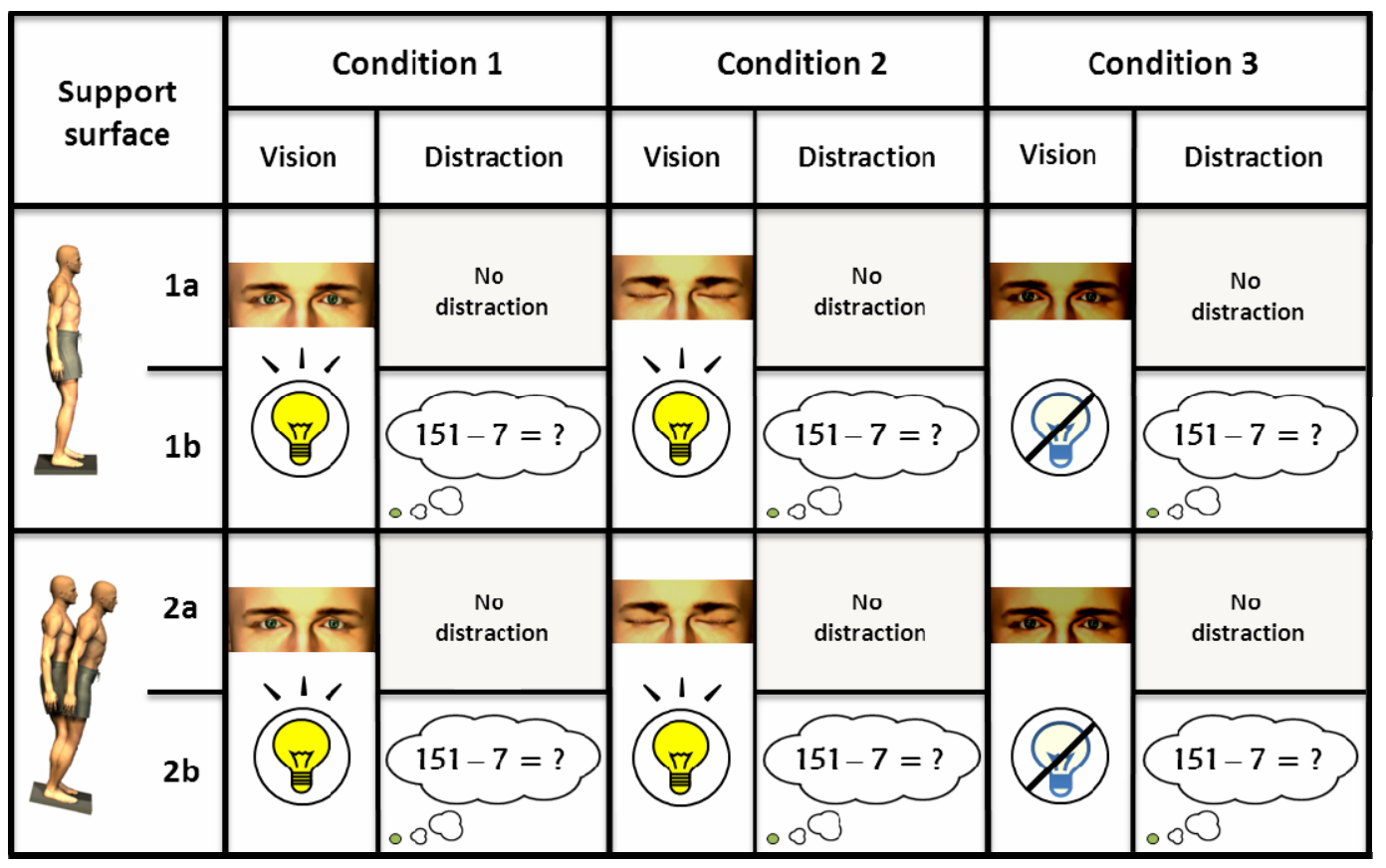

1-1a: EoOn, fixed surface, no distraction task; 1-1b: EoOn, fixed surface + distraction task (subtracting backwards aloud by 7 from a 3-digit number); 1-2a: EoOn, sway-referenced surface, no distraction task; 1-2b: EoOn, sway-referenced surface + distraction task; 2-1a: EcOn, fixed surface, no distraction task; 2-1b: EcOn, fixed surface + distraction task; 2-2a: EcOn, sway-referenced surface, no distraction task; 2-2b: EcOn, sway-referenced surface + distraction task; 3-1a: EoOff, fixed surface, no distraction task; 3-1b: EoOff, fixed surface + distraction task; 3-2a: EoOff, sway-referenced surface, no distraction task; 3-2b: EoOff, sway-referenced surface + distraction task

Figure 1. Illustration of the somatosensory (fixed and sway-referenced conditions), visual (eyes open in light or darkness and eyes closed conditions), and cognitive (no subtraction and subtraction conditions) test conditions.

score in each trial is:

$$
\mathrm{ES}=\left[12.5^{\circ}-\left(\theta_{\max }-\theta_{\min }\right)\right] / 12.5^{\circ} \times 100
$$

$12.5^{\circ}$ is the theoretical limit of postural stability along the sagittal plane $[21,22] . \theta_{\max }=$ peak forward sway angle about the ankle and $\theta_{\min }=$ peak backward sway angle (Figure 2). Performance in trials one and three were each analyzed with a 2 (Support) $\times 2$ (Distraction) $\times 3$ (Vision) repeated measures ANOVA. Speed and accuracy of sub- traction were analyzed with a 2 (Support) $\times$ 3 (Vision) repeated measures ANOVA (SAS statistical software (v. 9.1, SAS Institute Inc., North Carolina, USA). Significant interaction or main effects were followed up with 1-tailed simple effect comparisons. The test of significance for all analyses was set at $p<0.05$.

\section{RESULTS}

\subsection{Balance Control Performance}

In the first-trial analyses, the three-factor analysis of variance showed a significant main effect for Vision, $F(2$, $98)=98.4, \eta^{2}=0.66, p<0.0001$; a significant interaction effect between Vision and Distraction, $F(2,98)=$ $3.6, \eta^{2}=0.07, p=0.0351$; and a significant interaction effect between Vision and Support, $F(2,98)=52.0, \eta^{2}=$ $0.52, p<0.0001$. Subjects' peak-to-peak anteroposterior sway under the fixed surface condition with eyes open

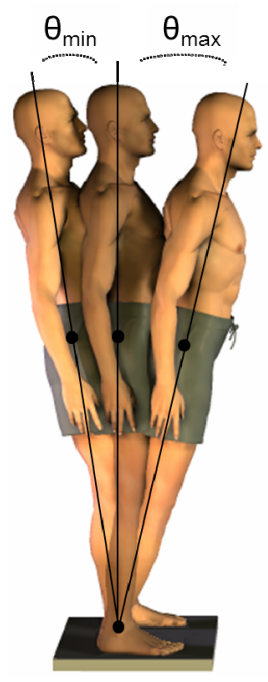

Figure 2. Quantification of the peakto-peak $\left(\theta_{\max }\right.$ to $\left.\theta_{\min }\right)$ anteroposterior sway. 
and lights on (EoOn) was 5\% less than with eyes closed (EcOn), $t(50)=11.0$, Cohen's $d=2.4, p<0.0001$ and $6 \%$ less than eyes open in the dark (EoOff), $t(50)=12.3$, $d=2.4, p<0.0001$. In the sway-referenced condition, EoOn was $24 \%$ less than EcOn, $t(50)=10.8, d=1.9, p<$ 0.0001 and $24 \%$ less than EoOff, $t(50)=10.5, d=2.1, p$ $<0.0001$. Sway between the EcOn and EoOff conditions did not differ from each other. Similar findings were observed when the subjects performed the concurrent subtraction task (Figure 3(a)).

Similar simple effects results were found in the thirdtrial analyses, in which the three-factor analysis of variance showed a significant main effect for Vision, $F(2,98)$ $=193.2, \eta^{2}=0.80, p<0.0001$; and a significant interaction effect between Vision and Support, $F(2,98)=93.5$,

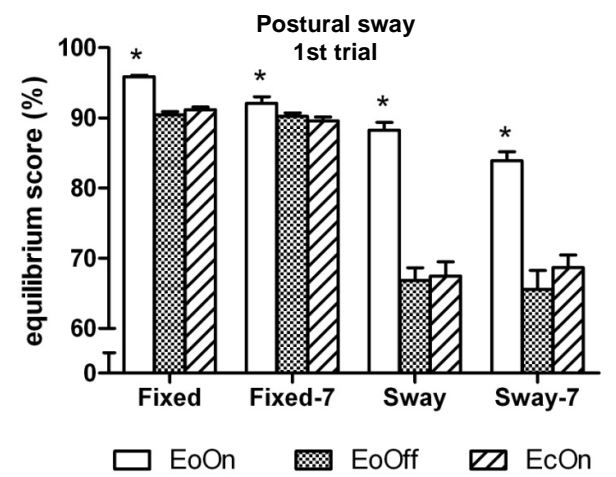

(a)

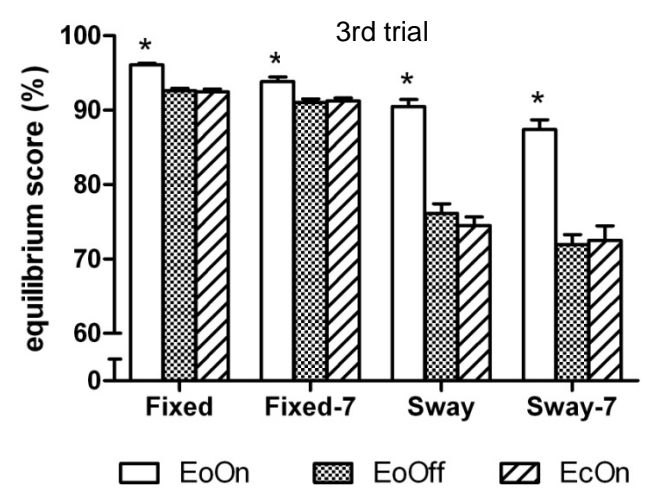

(b)

Figure 3. Mean peak-to-peak anteroposterior body center of mass sway in the (a) first and (b) third trial of each test condi- tion. Higher values indicate less sway. EoOn = eyes open with lights on; EoOff = eyes open in the dark; EcOn = eyes closed with lights on. Fixed $=$ standing on a stable (non-moving) sup- port surface; Fixed-7 = subtracting backwards by seven while standing on a fixed support surface; Sway = standing on a sup- port surface which moves in proportion to body sway; Sway-7 = subtracting backwards by seven while standing on a moving support surface. Error bars indicate one standard error. ${ }^{*} p<0.05$ compared to EoOff and EcOn. $\eta^{2}=0.66, p<0.0001$ (Figure 3(b)).

\subsection{Speed and Accuracy of Subtraction}

In the first-trial analyses, the two-factor analysis of variance showed a significant main effect for Vision, $F(2,98)$ $=6.9, \eta^{2}=0.12, p<0.0015$; and a significant main effect for Support, $F(1,49)=21.5, \eta^{2}=0.31, p<0.0001$. Subjects subtracted $6 \%$ faster with eyes closed compared to eyes open with lights on in the fixed surface condition, $t(50)=2.0, d=0.2, p=0.029$. They were 12 and $13 \%$ faster compared to eyes open with lights on and off in the sway-referenced support conditions, $t(50)=3.2, d=0.30$, $p=0.001$ and $t(50)=3.3, d=0.32, p=0.001$, respectively (Figure 4(a)). No difference in the speed of subtraction was observed among the conditions in the third trial (Figure 4(b)). Accuracy of subtraction was also similar among the conditions in the first and third trial (Figures 5(a) and (b)).

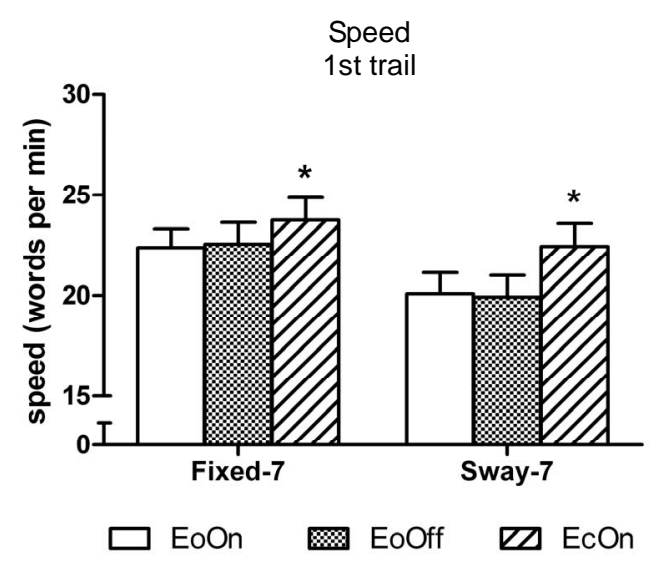

(a)

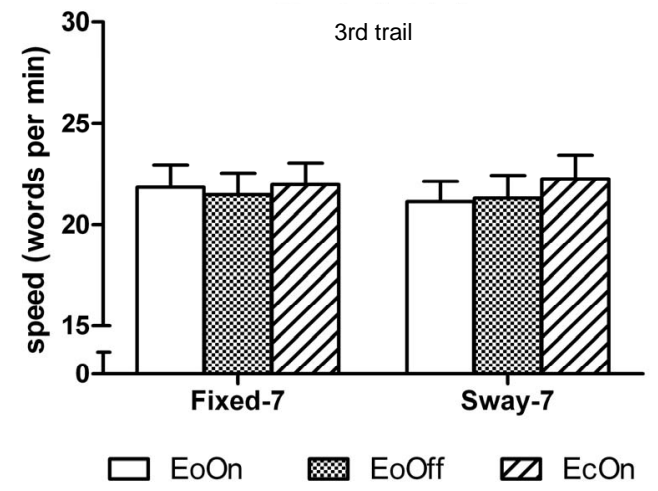

(b)

Figure 4. Mean speed of backward subtraction in the (a) first and (b) third trial of each test condition. Abbreviations are the same as Figure 2. Error bars indicate one standard error. ${ }^{*} p<0.05$ compared to EoOn in the fixed surface condition and compared to EoOff and EcOn in the sway-referenced surface condition. 


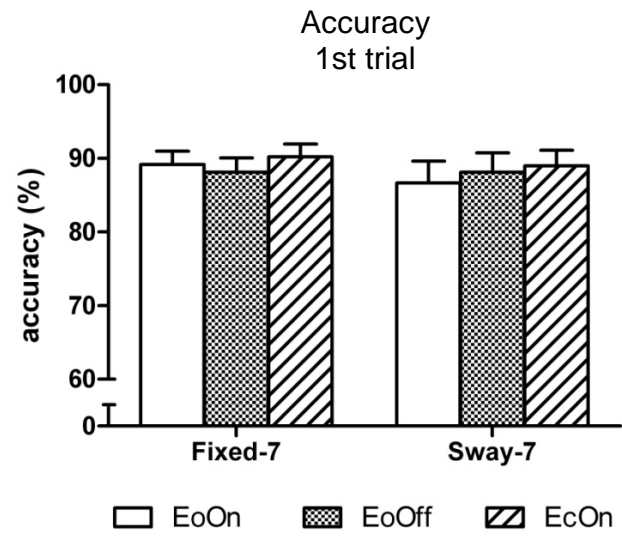

(a)

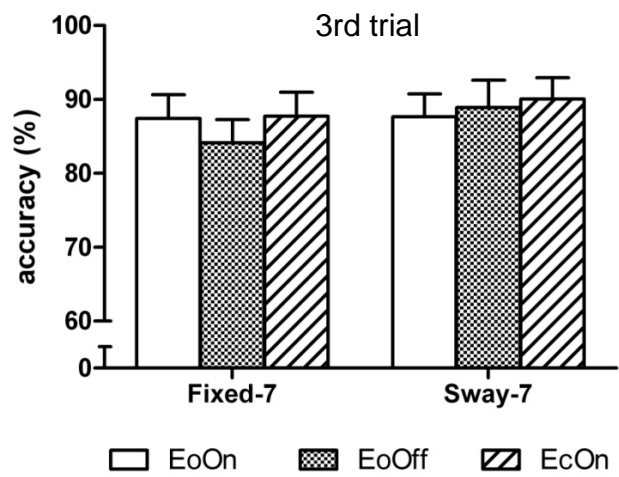

(b)

Figure 5. Mean accuracy of backward subtraction in the (a) first and (b) third trial of each test condition. There was no difference in accuracy among the test conditions.

\section{DISCUSSION}

\subsection{Specific Visuospatial Interference Effects of Dual-Tasking}

The results of the study revealed not only a task-difficulty component of the dual-task conditions but also the visuospatial interference between the postural and distraction tasks as predicted. The first component can be seen in the sway-referenced condition in which there is a sizeable decrease in balance control performance in the eyes closed and darkness conditions compared to performance in the fixed support surface condition. Similarly, the main effect of the support surface on the speed of subtraction also point to the increased difficulty in balance control from the fixed to sway-referenced condition. These outcomes are not surprising. The brain has less information to effectively integrate the senses needed for balance control. Visual inputs were not only absent with eye closure or open in the dark but somatosensory inputs were incongruent with vestibular information as well.

Of interest in our study, on the other hand, are the results which are consistent with our visuospatial interfer- ence hypothesis. In both support surface conditions, the speed of subtraction was reliably faster with eye closure compared to eyes open in normal lighting or total darkness. This finding is coherent with what was found previously [6] in that subtracting backwards during eye closure produced the least visuospatial interference with the balance control task.

Balance control under the sway-referencing condition remained compromised in the third trial of testing, but the normalization of the speed of subtraction suggests that some sort of adaptation may have occurred. It may involve the development of a more effective signal detection (selective attention) strategy [23], more efficient control of attention to plan and anticipate responses [24], and/or switching responses back and forth between the two tasks in which the execution of one task is withheld until the other task is underway [25]. These adaptations may $[6,26]$ or may not [27] include alterations in the frequency characteristics of postural sway.

\subsection{General Discussion and Application}

In this section we broaden the findings of this and the previous study [6] to potential clinical applications as well as the aviation domain in which one of us ( $\mathrm{SH}$ ) has particular expertise.

The results of the study suggest that a hierarchy of progression in balance control training conditions could be utilized. The incorporation of a secondary task into the rehabilitation program could progress in the following ascending order of difficulty:

1) Fixed surface with eyes open under typical lighting using a cognitive activity that does not unduly interfere with balance control, such as word generation [6];

2) Replace word generation with a subtraction task (retro-3 is easier, retro-7 is more difficult);

3) Fixed surface, eyes closed + word generation;

4) Replace word generation with a subtraction task;

5) Fixed surface, eyes open in the dark + word generation;

6) Replace word generation with a subtraction task;

7) Repeat 1-6 with sway-referenced surface;

8) Repeat 1-7 with sway-referenced surface and visual surround.

In the field of aviation, they relate specifically to situations experienced by pilots flying in poor visual conditions, where visual inputs outside the cockpit are no longer useful for spatial orientation. In these situations, pilots must instead rely on their instruments to maintain proper flight parameters and sense of postural orientation while ignoring the natural tendency to look out of the aircraft so as to avoid conflicts among the visual, somatosensory, and vestibular systems. These conflicts can produce spatial disorientation, the Coriolis illusion, and/ 
or vertigo, any of which can lead to fatal consequences. Sensory conflicts are most often caused by a high visuospatial processing load in which the external visual environment is incongruent with inputs from the somatosensory and vestibular systems, resulting in a mismatch with the body's internal frame of reference. These incongruent inputs are most often manifested as the visual system incorrectly signaling the aircraft in a turn while the vestibular system correctly senses the aircraft is straight and level.

In the current study, by attempting to discover if eyes open in the dark decreased balance control performance compared to eyes closed, we were essentially comparing the difference between a pilot maintaining spatial orientation while flying with night vision devices versus just instruments. We had hypothesized that, even in darkness, if the eyes are open, the visual centers will still try to draw in visual cues from the surrounding environment, which might conflict with somatosensory or vestibular inputs. This hypothesis is consistent with the experiences of pilots who become spatially disoriented while flying with night vision devices in the deserts of Iraq unless they turn off the night vision devices and focus their visual scans on their instruments.

The use of a distractive cognitive (Retro-7 subtraction) task to probe the same regions of the brain that are used to process visual inputs for balance control is also similar to the complex dynamics of aircraft control that pilots face when flying in degraded visual environments. Along this line of thinking, pilots are specifically trained to "stay ahead of the aircraft" in order to prevent "task fixation." A good example of this occurs when student pilots are asked to recite emergency procedures while flying an aircraft. As the student attempts to visualize the procedures which he/she has committed to memory, he/she is unable to maintain the assigned altitude and airspeed and ends up either too high and slow or too low and fast. In other instances, the pilot may be able to maintain his/her flight parameters but becomes unable to recite the emergency procedures quickly. The results of the current study suggest that task interference of the sorts described in aviation experiences occurs within the visuospatial processing area(s) of the brain. The ability to rapidly visualize and recite emergency procedures is being interfered with by the concomitant requirement to process visual inputs from the surrounding environment.

\section{REFERENCES}

[1] Keele, S.W. and Ivry, R. (1990) Does the cerebellum provide a common computation for diverse tasks? A timing hypothesis. Annals of the New York Academy of Sciences, 608, 179-207.

[2] Hayes, A.E., Davidson, M.C., Keele, S.W. and Rafal,
R.D. (1998) Toward a functional analysis of the basal ganglia. Journal of Cognitive Neuroscience, 10, 178-198. doi:10.1162/089892998562645

[3] Keele, S.W. (1981). Behavioral analysis of movement. In: Brooks, V., Ed., Handbook of Physiology: Sec. 1: The Nervous System, 2, Williams \& Wilkins, Baltimore, 1391-1414.

[4] Keele, S.W., Ivry, R.B. and Pokorny, R. (1987) Force control and its relation to timing. Journal of Motor Behavior, 19, 96-114.

[5] Keele, S.W. (1986). Motor control. In: Boff, K.R., Kaufman, L. and Thomas, J.P., Eds., Handbook of Perception and Human Performance, New York, John Wiley \& Sons, II, 1-60.

[6] Chong, R.K., Mills, B., Dailey, L., Lane, E., Smith, S. and Lee, K.H. (2010) Specific interference between a cognitive task and sensory organization for stance balance control in healthy young adults: Visuospatial effects. Neuropsychologia, 48, 2709-2718.

doi:10.1016/j.neuropsychologia.2010.05.018

[7] Chong, R.K., Ambrose, A., Carzoli, J., Hardison, L. and Jacobson, B. (2001) Source of improvement in balance control after a training program for ankle proprioception. Perceptual and Motor Skills, 92, 265-272. doi:10.2466/pms.2001.92.1.265

[8] Chong, R.K. and Franklin, M.E. (2001) Initial evidence for the mixing and soft assembly of the ankle, suspensory, and hip muscle patterns. Experimental Brain Research, 136, 250-255. doi:10.1007/s002210000578

[9] Chong, R.K., Chiu, F.C., Lee, K.H. and Do, M.C. (2009) An instance of reduced center of mass displacement: The Ba Gua Zhang walking gait. Perceptual and Motor Skills, 109, 646-648. doi:10.2466/pms.109.3.646-648

[10] Horak, F.B. (2006) Postural orientation and equilibrium: What do we need to know about neural control of balance to prevent falls? Age Ageing, 35, ii7-ii11. doi:10.1093/ageing/af1077

[11] Rueckert, L., Lange, N., Partiot, A., Appollonio, I., Litvan, I., Le Bihan, D., et al. (1996) Visualizing cortical activation during mental calculation with functional MRI. NeuroImage, 3, 97-103. doi:10.1006/nimg.1996.0011

[12] Galletti, C., Battaglini, P.P. and Fattori, P. (1995) Eye position influence on the parieto-occipital area PO (V6) of the macaque monkey. European Journal of Neuroscience, 7, 2486-2501. doi:10.1111/j.1460-9568.1995.tb01047.x

[13] Shipp, S., Blanton, M. and Zeki, S. (1998) A visuosomatomotor pathway through superior parietal cortex in the macaque monkey: Cortical connections of areas V6 and V6A. European Journal of Neuroscience, 10, 3171 3193. doi:10.1046/j.1460-9568.1998.00327.x

[14] Marx, E., Stephan, T., Nolte, A., Deutschlander, A., Seelos, K.C., Dieterich, M., et al. (2003) Eye closure in darkness animates sensory systems. NeuroImage, 19, 924-934. doi:10.1016/S1053-8119(03)00150-2

[15] Hufner, K., Stephan, T., Glasauer, S., Kalla, R., Riedel, E., Deutschlander, A., et al. (2008) Differences in saccade-evoked brain activation patterns with eyes open or 
eyes closed in complete darkness. Experimental Brain Research, 186, 419-430.

doi:10.1007/s00221-007-1247-y

[16] Tsubota, K., Kwong, K.K., Lee, T.Y., Nakamura, J. and Cheng, H.M. (1999) Functional MRI of brain activation by eye blinking. Experimental Eye Research, 69, 1-7. doi:10.1006/exer.1999.0660

[17] Kato, M. and Miyauchi, S. (2003) Functional MRI of brain activation evoked by intentional eye blinking. NeuroImage, 18, 749-759. doi:10.1016/S1053-8119(03)00005-3

[18] Chong, R.K., Jones, C.L. and Horak, F.B. (1999) Postural set for balance control is normal in Alzheimer's but not in Parkinson's disease. Journals of Gerontology Series A: Biological Sciences and Medical Sciences, 54, M129M135. doi:10.1093/gerona/54.3.M129

[19] Raichle, M.E. and Snyder, A.Z. (2007) A default mode of brain function: A brief history of an evolving idea. NeuroImage, 37, 1083-1090.

[20] Jamet, M., Deviterne, D., Gauchard, G.C., Vancon, G. and Perrin, P.P. (2004) Higher visual dependency increases balance control perturbation during cognitive task fulfillment in elderly people. Neuroscience Letters, 359, 61-64. doi:10.1016/j.neulet.2004.02.010

[21] Bronte-Stewart, H.M., Minn, A.Y., Rodrigues, K., Buckley, E.L. and Nashner, L.M. (2002) Postural instability in idiopathic Parkinson's disease: The role of medication and unilateral pallidotomy. Brain, 125, 2100-2114. doi:10.1093/brain/awf207

[22] McCollum, G. and Leen, T.K. (1989) Form and exploration of mechanical stability limits in erect stance. Journal of Motor Behavior, 21, 225-244.

[23] Sperling, G. (1984) A unified theory of attention and signal detection. In: Parasuraman, R. and Davies, D.R., Eds., Varieties of Attention, Academic Press, Inc., San Diego, 177-182.

[24] Rabbitt, P. (1984) The control of attention in visual search. In: Parasuraman, R. and Davies, D.R., Eds., Varieties of Attention, Academic Press, Inc., San Diego, 273-292.

[25] Meyer, D.E. and Kieras, D.E. (1997) A computational theory of executive cognitive processes and multiple-task performance. Part 1. Basic mechanisms. Psychological Review, 104, 3-65. doi:10.1037/0033-295X.104.1.3

[26] Williams, H.G., McClenaghan, B.A. and Dickerson, J. (1997) Spectral characteristics of postural control in elderly individuals. Archives of Physical Medicine and Rehabilitation, 78, 737-744. doi:10.1016/S0003-9993(97)90082-4

[27] Rougier, P. (2003) The influence of having the eyelids open or closed on undisturbed postural control. Neuroscience Research, 47, 73-83. doi:10.1016/S0168-0102(03)00187-1 